\title{
Frataxin deficiency in pancreatic islets causes diabetes due to loss of $\beta$ cell mass
}

\author{
Michael Ristow, ${ }^{1,2}$ Hindrik Mulder, ${ }^{3}$ Doreen Pomplun, ${ }^{1,2}$ Tim J. Schulz, ${ }^{1,2}$ \\ Katrin Müller-Schmehl, ${ }^{1}$ Anja Krause, ${ }^{1}$ Malin Fex, ${ }^{3}$ Hélène Puccio, ${ }^{4}$ Jörg Müller, ${ }^{5}$ \\ Frank Isken, ${ }^{1,2}$ Joachim Spranger, ${ }^{1,2}$ Dirk Müller-Wieland, ${ }^{6}$ Mark A. Magnuson, ${ }^{7}$ \\ Matthias Möhlig, ${ }^{1,2}$ Michel Koenig, ${ }^{4}$ and Andreas F.H. Pfeiffer ${ }^{1,2}$
}

${ }^{1}$ German Institute of Human Nutrition, Bergholz-Rehbrücke, Germany

${ }^{2}$ Free University of Berlin, Berlin, Germany

${ }^{3}$ Lund University, Lund, Sweden

${ }^{4}$ Institut de Génétique et de Biologie Moléculaire et Cellulaire, Strasbourg, France

${ }^{5}$ Magnettech GmbH, Berlin, Germany

${ }^{6}$ German Diabetes Research Institute, Düsseldorf, Germany

${ }^{7}$ Vanderbilt University, Nashville, Tennessee, USA

\begin{abstract}
Diabetes is caused by an absolute (type 1 ) or relative (type 2 ) deficiency of insulin-producing $\beta$ cells. We have disrupted expression of the mitochondrial protein frataxin selectively in pancreatic $\beta$ cells. Mice were born healthy but subsequently developed impaired glucose tolerance progressing to overt diabetes mellitus. These observations were explained by impairment of insulin secretion due to a loss of $\beta$ cell mass in knockout animals. This phenotype was preceded by elevated levels of reactive oxygen species in knockout islets, an increased frequency of apoptosis, and a decreased number of proliferating $\beta$ cells. Hence, disruption of the frataxin gene in pancreatic $\beta$ cells causes diabetes following cellular growth arrest and apoptosis, paralleled by an increase in reactive oxygen species in islets. These observations might provide insight into the deterioration of $\beta$ cell function observed in different subtypes of diabetes in humans.
\end{abstract}

J. Clin. Invest. 112:527-534 (2003). doi:10.1172/JCI200318107.

\section{Introduction}

Friedreich ataxia (FA) is an autosomal-recessively inherited disease that leads to neurodegeneration (1) as well as cardiomyopathy, causing premature death at an average age of 37 years (2). In most cases, FA is caused by an intronic GAA triplet repeat expansion (3) that impairs expression levels of a fully functional protein named frataxin (4). Frataxin in its mature state is an $18-\mathrm{kDa}$ protein encoded in the nucleus and located at the mitochondrial matrix (2). It has been previously demonstrated that this protein directs iron-sulfur-cluster assembly (5), hereby affecting oxidative energy flux (6).

Received for publication February 12, 2003, and accepted in revised form June 10, 2003.

Address correspondence to: Michael Ristow, German Institute of Human Nutrition, Department Klinische Ernährung, 114 Arthur-Scheunert-Strasse, D-14558 Bergholz-Rehbrücke, Germany. Phone: 49-33200-88-787; Fax: 49-33200-88-777; E-mail: mristow@mristow.org.

Hindrik Mulder, Doreen Pomplun, Tim J. Schulz, Katrin Müller-Schmehl, and Anja Krause contributed equally to this work.

Conflict of interest: The authors have declared that no conflict of interest exists.

Nonstandard abbreviations used: Friedreich ataxia (FA); reactive oxygen species (ROS); electron spin resonance (ESR); 2,2-dimethyl-1-pyrroline- $N$-oxide (DMPO); milliTesla (mT); 4-hydroxy-2,2,6,6-tetramethyl-piperidine-1-oxyl (TEMPOL); $2^{\prime} 7^{\prime}$-di-chloro-fluorescein (DCF); 2'7'-di-hydro-chlorofluorescein-di-acetate $\left(\mathrm{H}_{2} \mathrm{DCF}\right)$.
A wide body of evidence suggests a role for frataxin in promoting cellular defense against reactive oxygen species (ROS): clinical data demonstrate increased oxidative stress in patients with FA $(7,8)$. Findings from animal models suggest that, while the nonconditional knockout is embryonically lethal (9), the tissue-specific targeted disruption of the frataxin gene in heart or neuronal tissues causes depletion of iron-sulfur clusters, presumably accompanied by ROS formation (10). In vitro findings indicate that frataxindependent accumulation of unspecified ROS is dependent on the concurrent reduction of superoxide dismutase activity in frataxin-deficient states (11) and elevation of glutathione peroxidase activity in frataxin-overexpressing states (12).

Despite their severely reduced life expectancy, a subset of FA patients develops diabetes mellitus of unknown origin (13). Nondiabetic FA patients exhibit normal basal insulin oscillations (M. Ristow et al., unpublished observations) and normal glucose-stimulated insulin secretion (13) but show some degree of insulin resistance $(14,15)$. While studies on a possible association between the common type 2 diabetes and the frataxin GAA triplet repeat expansions in humans are inconclusive (16-21), linkage of type 2 diabetes with the locus harboring the human frataxin gene at $9 \mathrm{q} 13$ was found in at least four different populations worldwide (22-25), suggesting a role in the pathogenesis of common type 2 diabetes. 
To functionally elucidate this suggestive link between frataxin deficiency and type 2 diabetes mellitus, we have disrupted the frataxin gene specifically in pancreatic $\beta$ cells of C57BL/ 6 mice using the cre/loxP technique (26). The lack of frataxin protein in pancreatic islets caused a reduction of insulin-secretory capacity, resulting in overt diabetes mellitus in older animals.

\section{Methods}

Animals. Mice were housed in standard barrier facilities according to Federation of European Laboratory Animal Science Associations regulations and were fed standard chow (Altromin GmbH, Lage, Germany). Ins2-cre founders were reported to be $66 \% \mathrm{C} 57 \mathrm{BL} / 6$ initially and were backcrossed with $\mathrm{C} 57 \mathrm{BL} / 6$ three times prior to breeding of the study group. LoxP founders were reported to be $50 \% \mathrm{C} 57 \mathrm{BL} / 6$ initially and were backcrossed with $\mathrm{C} 57 \mathrm{BL} / 6$ four times prior to breeding of the study group. Hence, genetic background of the intercrosses was $92.5 \%$ C57BL/6 minimum. All animal experiments were approved by the governmental animal-ethics committee. For BrdU analyses, mice were injected with $50 \mathrm{mg} / \mathrm{kg}$ body weight BrdU (SigmaAldrich, Munich, Germany) 3 hours before sacrifice.

Genotype analyses. Genomic PCR for detection of the loxP allele was performed as previously described (10). Genomic PCR for detection of the Cre transgene was performed using primers 5'-TCTCACGTACTGACGGTGG and 5'-ACCAGCTTGCATGATCTCC for 35 cycles at an annealing temperature of $70^{\circ} \mathrm{C}$ located within the cDNA of the Cre transgene. Genomic PCR for detection of the knockout allele (Figure 1a) was performed as previously described (9) using primers $5^{\prime}$ CTGTTTACCATGGCTGAGATCTC and 5'-GTAGCTGGCTGGGAAATGAA, located in the introns flanking exon 4 of the frataxin gene upstream and downstream of this exon. Reversely transcribed PCR for detection of the knockout transcript (Figure 1b) was performed as previously described (10) using primers $5^{\prime}$-CACTTGGATCCTCTAGACGAGACAGCG and 5'-TTTAGTCAGCTCCCTGGCC, located in exon 3 and exon 5 of the frataxin cDNA, respectively. DNA size markers for all experiments were the 1-kb DNA ladder (15615-024; Invitrogen, Karlsruhe, Germany).

Physiological analyses. For all experiments, in addition to the knockout genotype, three control genotypes were evaluated: homozygous loxP animals, heterozygous Ins2cre animals, and wild-type animals carrying neither of the genetic modifications. Fasting and random-fed blood glucose was determined from tail vein bleeds and measured using MediSense Precision Plus Electrodes (Abbott GmbH \& Co. KG, Wiesbaden, Germany). A minimum of ten animals per genotype was evaluated (40 total for all genotypes). Glucose-tolerance tests were performed as previously described (27). A minimum of six animals per genotype was evaluated (24 total for all genotypes). Insulin-tolerance tests were performed as previously described (27). A minimum of six animals per genotype was evaluated (24 total for all genotypes). $\beta$-hydroxybutyrate levels were determined using MediSense Beta-Ketone Electrodes (Abbott GmbH \& Co. KG). A minimum of six animals per genotype was evaluated (24 total for all genotypes). Mouse serum insulin levels were determined by ELISA for rat insulin using a mouse insulin standard (both from CrystalChem Inc., Chicago, Illinois, USA). A minimum of six animals per genotype was evaluated (24 total for all genotypes).

Histology, immunohistochemistry, and islet morphology. Histology and immunohistochemistry were performed using standard procedures. Primary antibodies used were anti-insulin (Camon, Wiesbaden, Germany), antiglucagon, anti-somatostatin (both DAKO A/S, Glostrup, Denmark), anti-murine frataxin (10), antiKi67 (Dianova, Hamburg, Germany), anti-BrdU (Roche Molecular Biochemicals, Mannheim, Germany), and against activated caspase-3 (Pharmingen, Hamburg, Germany). Islet morphometry was performed on six non-consecutive longitudinal sections of the pancreas of three animals per genotype of the same sex using a digital microscope (Eclipse E1000; Nikon Deutschland $\mathrm{GmbH}$, Düsseldorf, Germany) and quantification software (Lucia G version 4.61; Nikon Deutschland GmbH), evaluating 18 sections per genotype. Quantification of apoptotic and proliferating cells was performed on the ten largest islets per section of 18 sections derived from three animals of each genotype (i.e., 60 islets per animal).

Isolation and static incubation of islets. Islets were isolated by retrograde injection of collagenase (Roche Molecular Biochemicals) into the pancreatic duct according to standard procedures (28). For secretion studies, batches $(n=6-8)$ of three islets for each condition were kept in HBSS (125 mM NaCl, $5.9 \mathrm{mM} \mathrm{KCl}, 1.2$ $\mathrm{mM} \mathrm{MgCl}_{2}, 20 \mathrm{mM}$ HEPES, $1.3 \mathrm{mM} \mathrm{CaCl}_{2}, 0.1 \% \mathrm{BSA}$; $\mathrm{pH} 7.35$ ) containing $2.8 \mathrm{mM}$ glucose for 60 minutes in an incubator at $37^{\circ} \mathrm{C}$. Then, three islets at a time were transferred to a multiwell plate kept on ice and containing $200 \mu \mathrm{l}$ per well of the same buffer but with the addition of the respective secretagogue (see Figure 3 ). When all islets had been transferred, the plate was again placed in an incubator at $37^{\circ} \mathrm{C}$; after $60 \mathrm{~min}$ utes, a sample from the buffer was removed for measurement of insulin by ELISA. Islet insulin content was measured following extraction by acidic ethanol and subsequent ELISA for mouse insulin (see Physiological analyses, above) normalized for protein content (29).

Detection of reactive oxygen species. Electron spin resonance (ESR) analyses in islets were performed in buffer consisting of 8\% RPMI 1640, 2\% FBS, 1\% DMSO, and 89\% Hanks' medium (all from SigmaAldrich), containing $45 \mathrm{mM}$ 2,2-dimethyl-1-pyrroline$N$-oxide (DMPO; Alexis Deutschland GmbH, Grünberg, Germany) as a spin trap. After incubation for 45 minutes at $37^{\circ} \mathrm{C}$, ESR spectra were recorded on a MiniScope MS100 spectrometer (Magnettech GmbH, Berlin, Germany) using the following parameters: 0.22 milliTesla (mT) modulation amplitude, $20 \mathrm{~mW}$ microwave power, 60 seconds sweep time, and 16 accumulation cycles. As a positive control, a mixture 
$\mathbf{a}$

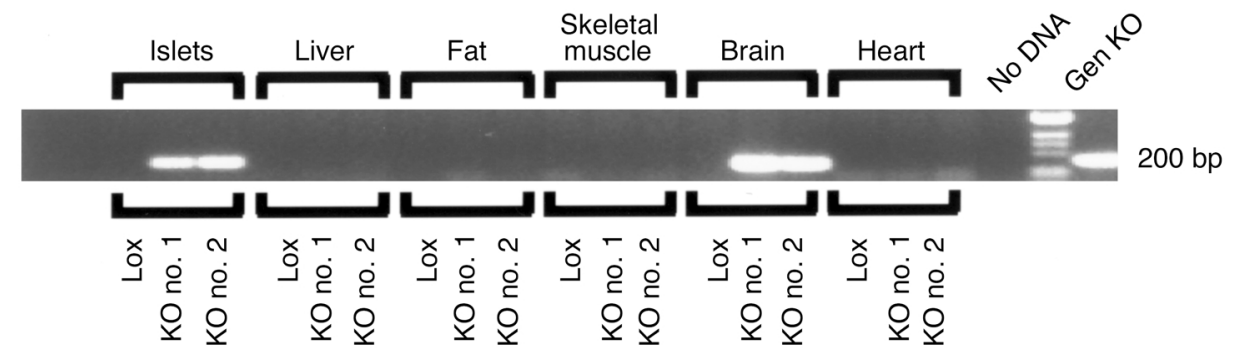

b
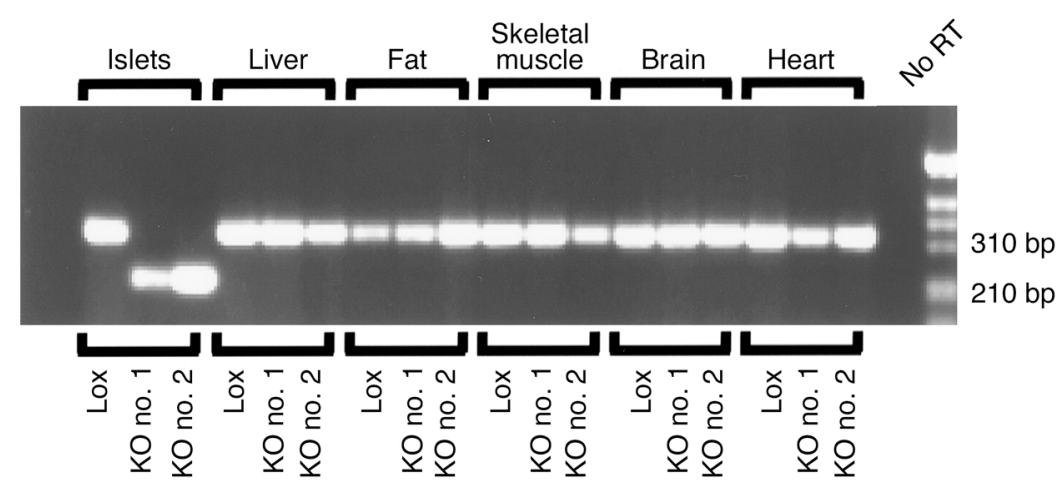

C

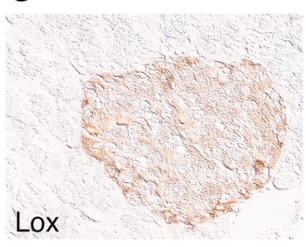

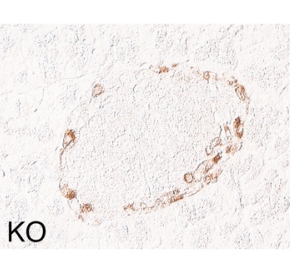

d

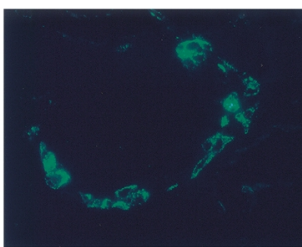

Anti-glucagon

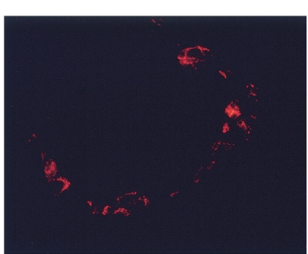

Anti-frataxin

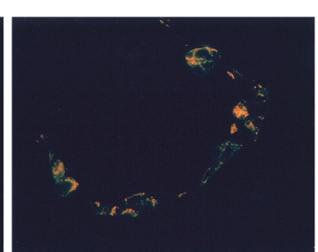

Merge

\section{Figure 1}

Tissue-specific knockout of the frataxin gene in pancreatic $\beta$ cells. (a) A genomic PCR derived from primers located up-and downstream of the putatively knocked-out exon 4 of the frataxin gene. DNA was isolated from indicated tissue from one 4-week-old lox control animal (first lane of each triplet) and two 4-week-old knockout animals (second and third lanes of each triplet). DNA from a whole-body knockout (Gen $\mathrm{KO}$ ) was used as a positive control (9). (b) A reversely transcribed PCR using RNA from the same animals as in a, using primers located in exons 3 and 5 of the murine frataxin cDNA. (c) Subsets of microphotographs (original magnification, $\times 200$ ) of immunohistochemistry against murine frataxin on islets from 4-week-old animals of the indicated genotype. (d) Microphotographs (original magnification, $\times 200$ ) of one representative knockout islet immunohistochemically stained for glucagon (green, left) and frataxin (red, middle). Overlay of both microphotographs indicates colocalization of the remaining frataxin signals in knockout islets with glucagon-positive cells.

of hypoxanthine $(500 \mu \mathrm{M})$, xanthine oxidase $(5 \mathrm{U} / \mathrm{ml})$ (both from Sigma), and the spin trap DMPO (45 mM) was used to generate a standard superoxide signal. The detection limit of trapped superoxide was calculated against a chemically defined 4-hydroxy-2,2,6,6-tetramethyl-piperidine-1-oxyl (TEMPOL; Alexis Deutschland) standard solution at a concentration of $1 \mu \mathrm{M}$. Identification of superoxide as the type of ROS present was performed through comparison with its standard coupling coefficients: $\mathrm{A}\left(\mathrm{H}_{\beta}\right)=1.144 \mathrm{mT}$ and $\mathrm{A}(\mathrm{N})=1.4303 \mathrm{mT}$. Peak and trough points in Figure $4 \mathrm{a}$ indicate the peaks and troughs of the spectrum that belong to the superoxide signal.

$2^{\prime} 7^{\prime}$-di-chloro-fluorescein-based (DCF-based) detection of unspecified ROS was performed as previously described (12) by loading islets with the nonfluorescent, reduced form of DCF, 2'7'-di-hydro-chlorofluorescein-di-acetate $\left(\mathrm{H}_{2} \mathrm{DCF}\right)$ (Molecular Probes Europe BV, Leiden, The Netherlands), which is oxidized to
DCF-di-acetate in the presence of ROS. Fluorescence was exited at $488 \mathrm{nM}$ (Eclipse E1000; Nikon).

Statistical analyses. All calculations were performed with SPSS version 8.0.0 (SPSS Science, Chicago, Illinois, USA) software. Mendelian distribution of offspring was evaluated by $\chi^{2}$ analysis. All other significances were tested by independent $t$ tests. Equality of distribution was tested by Levine's test. $P$ values were considered significant at less than 0.05 . Significance of knockout versus control groups was calculated against combined controls (consisting of wild-type, heterozygous Ins2-cre, and homozygous loxP animals) as well as each genotype separately. Significance was assumed when all four calculations reached significance independently, i.e., only when knockout measurements were significantly different from those of homozygous loxP animals, heterozygous Ins2-cre animals, and wild-type animals as determined in separate calculations. Hence, in the unlikely event that one (or 
more) of the four calculations (knockout vs. loxP, knockout vs. Ins2-cre, knockout vs. wild-type, and knockout vs. all three combined) was shown to be insignificant, overall results were indicated as not significant. Using this approach, possible unwanted influences of genetic alterations within Ins2-cre animals or loxP animals on the phenotype described for the knockout animals were minimized.

\section{Results}

Disruption of the frataxin gene in pancreatic $\beta$ cells. Intercrosses of mice carrying the loxP allele (10) as well as mice transgenically expressing Cre recombinase under the control of the rat insulin promoter 2 (Ins2) [C57BL/6-TgN(Ins2Cre)25Mgn] $(27,30,31)$ were employed to generate a $\beta$ cell-specific knockout of the frataxin gene. Breeding of animals heterozygous for the frataxin loxP allele and either paternally or maternally heterozygous for Ins2-cre generated $11.7 \%$ knockout animals (expected frequency 12.5\%) defined by homozygosity for the loxP allele and heterozygosity for the Ins2-cre allele as determined by genomic PCR from tail biopsies. From 71 pregnancies, 572 offspring were derived. Frequencies of all remaining genotypes followed mendelian distribution (total of expected vs. obtained genotypes: $P=0.838$ ).

To examine the presence and tissue-specificity of the knockout, removal of exon 4 from genomic frataxin DNA was demonstrated by genomic PCR (Figure 1a). As previously described (31), the Ins2 promoter becomes transiently activated during embryonic development in subregions of the mouse brain, as indicated by the genomic signal derived from brain DNA (Figure 1a). To investigate the transcriptional product, reversely transcribed PCR employing primers located in the flanking exons 3 and 5 was applied to RNA extracts from respective tissues. The shortened transcript of a knocked-out frataxin gene was detected in pancreatic islets of Langerhans only, while those parts of the brain carrying knocked-out genomic frataxin DNA did not transcribe the gene (Figure 1b). Finally, efficacy of the knockout in $\beta$ cells was demonstrated by immunohistochemistry (Figure 1c): $\beta$ cells, primarily located within the center of pancreatic islets, lacked detectable frataxin protein in knockout animals. To further investigate the remnant signals at the periphery of knockout islets, these were identified to be immunopositive for both glucagon and frataxin (Figure 1d), suggesting that those islet cells that stained positive for frataxin in knockout animals are indeed $\alpha$ cells. Consistent with previously published findings in the muscle-specific frataxin knockout (10), we generally did not observe remnant wild-type signals by RT-PCR within the knockout tissue (Figure 1b). However, frataxin was detectable in $\alpha$ cells by immunohistochemistry (Figure $1 \mathrm{~d}$ ), presumably reflecting a preferential amplification of the shorter fragment, which was previously found to be typical for shortened internal standards employed in semiquantitative PCR reactions (32).

Together, the cre/loxP system efficiently suppressed expression of frataxin protein specifically in pancreatic $\beta$ cells. To study the effects of the $\beta$ cell disruption of frataxin, in vivo tests were applied to evaluate the physiological role of the protein in regard to glucose metabolism.

Disruption of frataxin causes diabetes at higher age. To test our initial hypothesis that deficiency of frataxin might cause diabetes mellitus in mice, random-fed (Figure 2a) and fasting (Figure 2b) blood glucose levels were determined. Random-fed glucose levels were significantly elevated starting at an age of 2 months (Figure 2a), reflecting impaired glucose tolerance in these animals. While this postprandial effect was observed in comparably young animals, fasting serum glucose levels became diabetic not earlier than at an age of 9 months (Figure 2b). The reasons for decreased postprandial glucose levels in very young animals remain to be evaluated
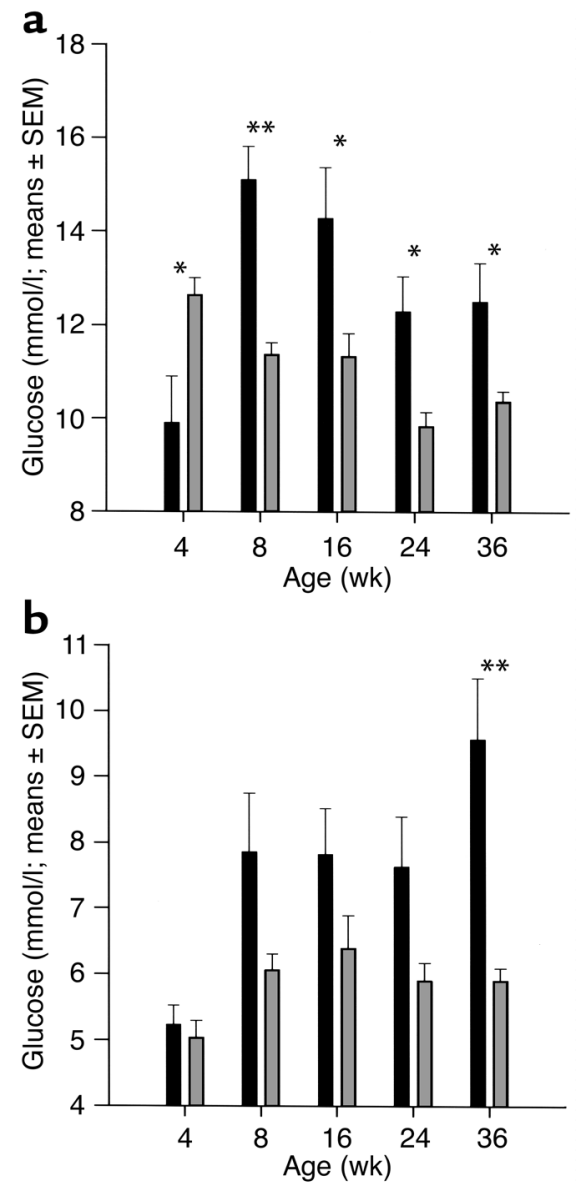

Figure 2

Diabetes develops progressively in $\beta$ cell-specific frataxin knockout animals. (a) Blood glucose concentrations in knockout animals (indicated by black bars) and control animals (comprising wild-type, heterozygous Ins2-cre, and homozygous loxP genotypes; indicated by gray bars) after overnight ad libitum supply of standard chow (randomfed). (b) Blood glucose concentrations after an overnight fast. Error bars depict SEM. ${ }^{*} 0.05>P>0.005 ;{ }^{*} P<0.005$. 
a
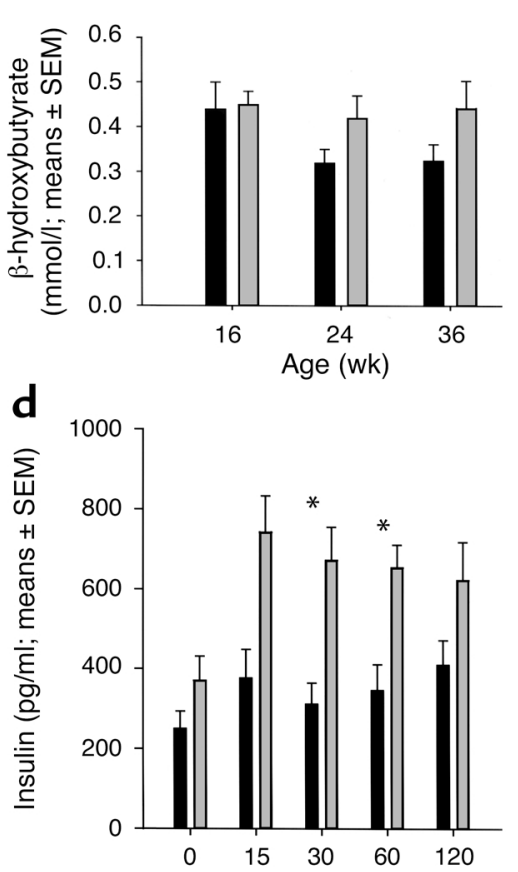

Time after i.p. glucose injection ( $\mathrm{min}$ )

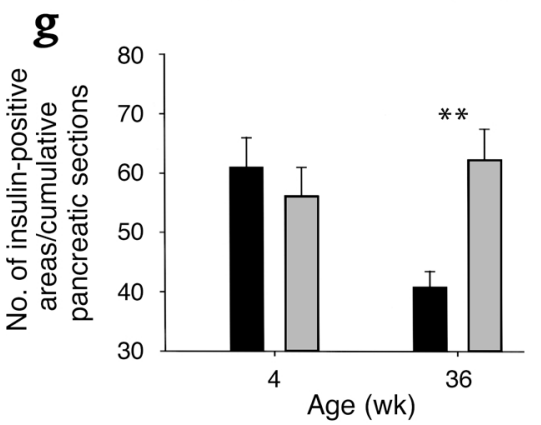

b

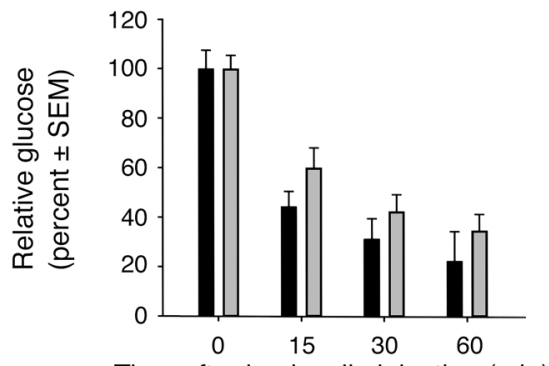

Time after i.p. insulin injection (min)

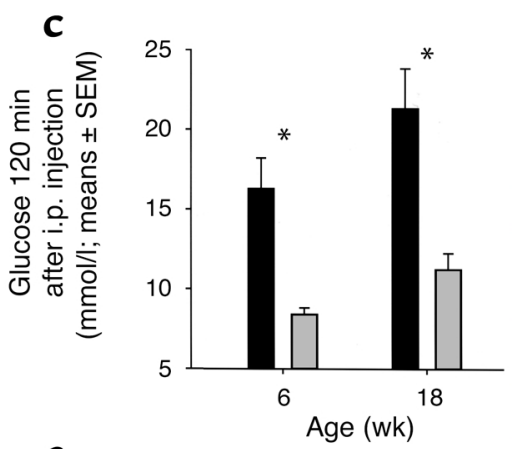

e

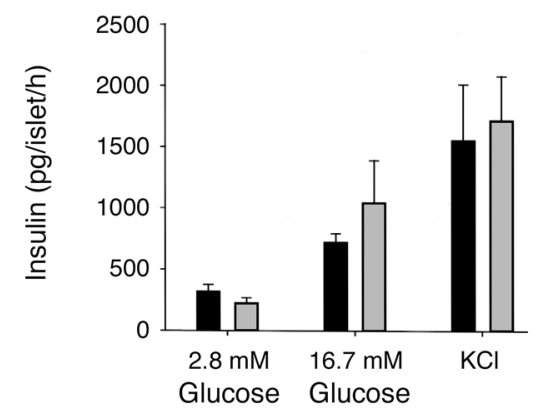

h

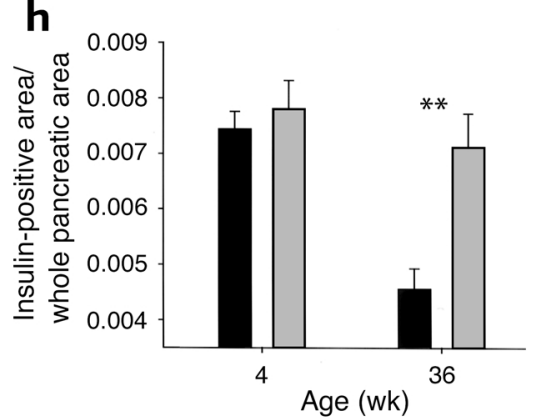

i
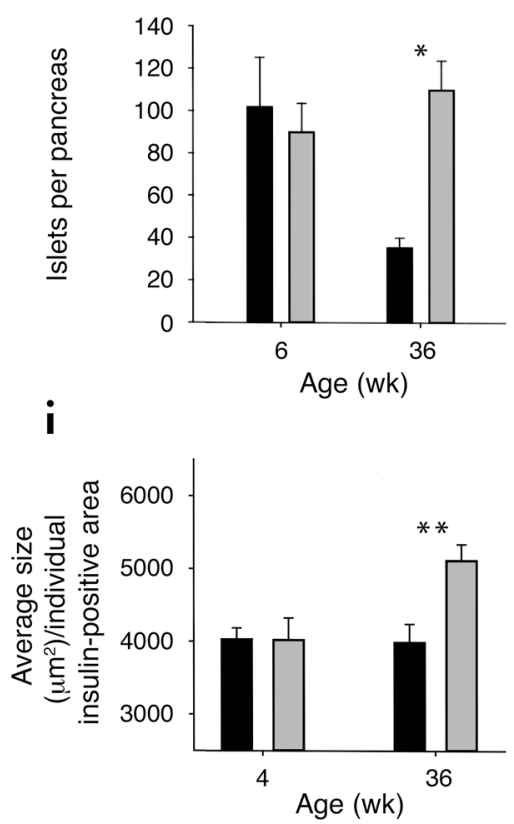

j 4 weeks

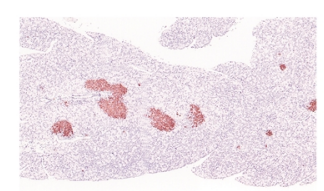

Lox

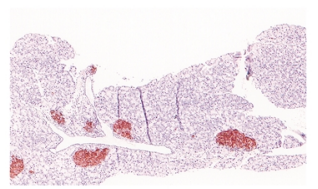

KO

36 weeks

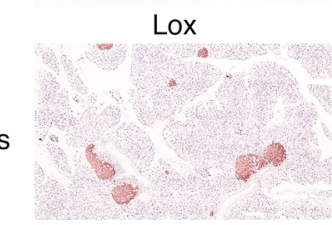

\section{Figure 3}

Diabetes develops because of decreased insulin secretion caused by a progressive reduction in $\beta$ cell mass. (a) $\beta$-hydroxybutyrate levels in knockout animals at different ages. (b) Results of an insulin-tolerance test at an age of 18 weeks. (c) Blood glucose levels 2 hours after intraperitoneal (i.p.) injection of glucose. (d) Insulin levels after intraperitoneal injection of glucose in 18-week-old mice. (e) Static incubations of isolated islets from 6-week-old animals in $35 \mathrm{mM}$ potassium chloride and different concentrations of glucose (as indicated). (f) Number of islets isolated by collagenase digestion from pancreata of mice at different ages. (g) Number of insulin-positive areas on all pancreatic sections per pancreas from mice at different ages. (h) Ratios of total insulin-positive area in pancreata from mice at different ages per whole pancreatic area. (i) Average size per insulin-positive area from mice at different ages. Black bars, knockout animals; gray bars, control animals. (j) Subsets of microphotographs (original magnification, $\times 40$ ) from mice evaluated in $g$, using an anti-insulin antibody. Error bars depict SEM. ${ }^{*} 0.05>P>0.005 ;{ }^{*} P<0.005$.

(Figure 2a, 4 weeks). Taken together, the data suggest that lack of frataxin in pancreatic $\beta$ cells of mice leads to impaired glucose tolerance in adolescence, cumulat- ing in overt diabetes in midlife. This phenotype can be attributed to several possible dysfunctions of glucose metabolism, which were subsequently evaluated. 
Frataxin-deficient diabetes due to impaired insulin secretion. To elucidate the cause for this delayed-onset diabetes due to lack of frataxin protein, knockout mice were investigated in regard to possible insulin resistance, and absolute or relative insulin deficiency, all known to cause glucose intolerance and diabetes. Insulin levels were not significantly different in the fasting state (not shown for every age; e.g., at 3 months of age, $253.5 \pm 43.98 \mathrm{pg} / \mathrm{ml}$ for knockout animals and $332.1 \pm 38.52 \mathrm{pg} / \mathrm{ml}$ for control animals, $P=0.193$ ); neither did we find evidence for ketotic lipolysis, since body weight was normal (not shown for every age; e.g., at 3 months of age, $24.5 \pm 1.35$ $\mathrm{g}$ for knockout animals and $25.7 \pm 0.79 \mathrm{~g}$ for control animals, $P=0.449$ ) and since levels of $\beta$-hydroxybutyrate were normal at every age examined (Figure 3a). Together, these findings rule out the possibility of absolute insulin deficiency. Insulin sensitivity was determined by intraperitoneal injection of insulin and showed no significant difference between knockout and control animals (Figure $3 \mathrm{~b}$ ), suggesting that the cause for the diabetes observed in these animals might be located within the $\beta$ cell. Indeed, when animals were intraperitoneally challenged with glucose, knockout animals showed a significantly decreased glucose clearance rate up to 2 hours after the challenge (Figure 3c). Concurrently, we observed an increased area under the curve for serum glucose in knockout animals after a challenge to the sugar ( 6 weeks, $P=0.0647 ; 18$ weeks, $P=0.015)$. These observations were explained by an insufficient insulin-secretory response after glucose challenge in knockout animals (Figure 3d). Taken together, the results indicate that diabetes in $\beta$ cell-specific frataxin knockout animals occurs because of impaired insulin secretion rather than insulin resistance.

Impaired insulin secretion due to reduced $\beta$ cell mass and number. The underlying mechanisms for insufficient insulin secretion are known to be either a reduction of secretory capacity or an impairment of stimulussecretion coupling. Since nondiabetic FA patients exhibit normal glucose-stimulated insulin secretion and normal basal oscillations (see Introduction), an impaired stimulus-secretion coupling appeared unlikely. Nevertheless, static incubations of islets isolated

\section{Figure 4}

Increased levels of ROS, increased apoptosis, and decreased proliferation precede reduction of $\beta$ cell mass. (a) Readouts from ESRbased detection of superoxide in control islets (upper panel) and knockout islets (lower panel) from 6-week-old animals. Gray lines depict the raw signal with magnetic fields, and black lines depict the filtered signal. Circles indicate peaks and troughs used to determine the type of ROS, in this case specifically indicating the presence of superoxide. a.u., arbitrary units. (b) Fluorescence at $488 \mathrm{~nm}$ after loading of islets with $\mathrm{H}_{2} \mathrm{DCF}$, showing higher levels of DCF, an oxidation product of $\mathrm{H}_{2} \mathrm{DCF}$, in knockout islets in the presence of $11.1 \mathrm{mM}$ glucose. Insets depict native islets. (c) Quantification of islet cells immunohistochemically positive for activated caspase-3, a marker of apoptosis, as well as Ki67 and BrdU, both markers for proliferation. Black bars, knockout animals; gray bars, control animals; error bars, SEM. ${ }^{*} P<0.005$. from 6-week-old animals were performed. Insulin secretion from isolated, size-matched islets (see below, Figure 3i) did not reveal any significant differences in response to either low or high glucose concentrations or potassium chloride (Figure 3e). Furthermore, insulin content in isolated islets was found to be similar in both affected and nonaffected mice (at 6 weeks of age, $54.9 \pm 3.89 \mathrm{ng} / \mu \mathrm{g}$ protein for knockout animals and $60.3 \pm 3.84 \mathrm{ng} / \mathrm{ug}$ protein for control animals, $P=0.454$ ). Subsequently, insulin-secretory capacity in affected and nonaffected mice was determined using two independent techniques. First we quantified the number of islets in knockout compared with control animals by collagenase digestion of the pancreas. The number of islets was identical in young animals but became strikingly different with increasing age (Figure 3f). These findings were confirmed on sections from whole pancreata employing immunohistochemical methods. Here, a reduction of the overall insulin-positive area,

a
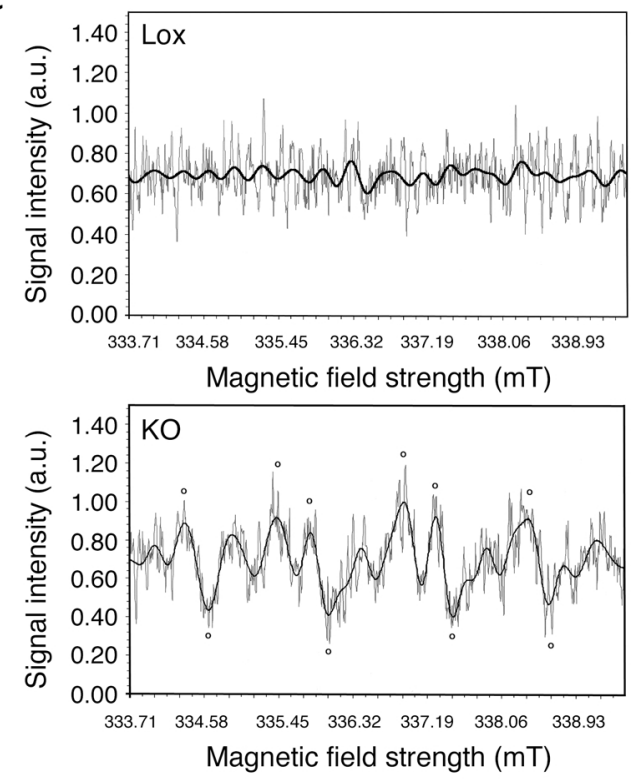

b
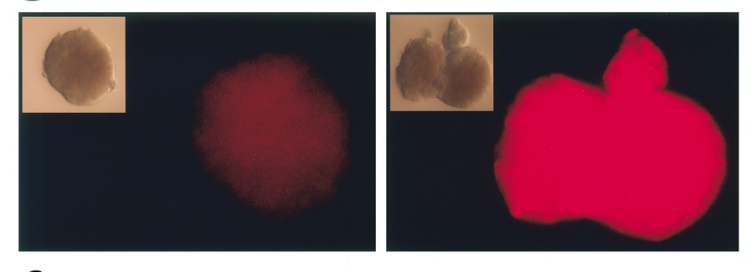

C

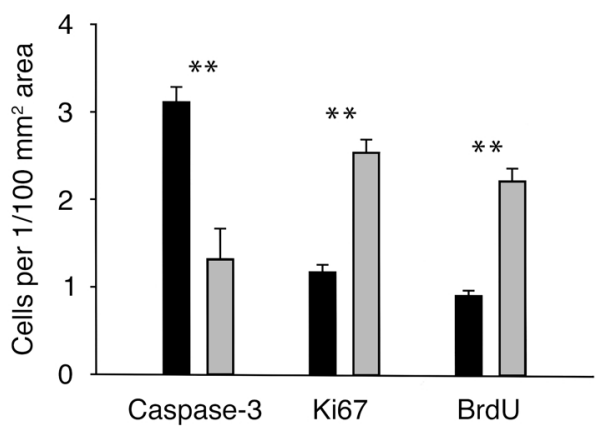


reflecting $\beta$ cell mass, was found in knockout animals of higher age (Figure $3, g-j$ ), while islet number and $\beta$ cell mass in young animals were unaltered (Figure $3, \mathrm{~g}-\mathrm{j}$ ). Specifically, we observed a reduced frequency of insulinpositive areas (reflecting islet number; Figure 3g), a reduction of overall insulin-positive area per pancreatic area (Figure 3, h and $\mathrm{j}$ ), and a relative reduction of islet size (Figure 3i) in older knockout animals only. Taken together, the data suggest that mice with $\beta$ cell-specific disruption of the frataxin gene become diabetic with increasing age because of a decrease in $\beta$ cell mass, islet number, and relative islet size, indicating a severely reduced insulin-secretory capacity in affected animals.

Disruption of frataxin causes free radical overload in pancreatic islets. Since lack of frataxin in humans leads to increased serum markers of oxidative stress, we questioned whether the observed decrease in insulin-secretory capacity, reflecting a progressively degenerative phenotype, might be accompanied by increased formation of ROS within islets of frataxin knockout mice. Hence, formation of ROS within isolated pancreatic $\beta$ cells was determined using two independent methods. Firstly, we employed the ESR technique after adding the spin-trap reagent DMPO to capture free radical formation. This method revealed detectable levels of trapped superoxide in knockout islets of 6-week-old animals only, while this anion was below detection limit for trapped radicals ( $50 \mathrm{nM}$ as determined by a chemically defined oxidated spin-trap, TEMPOL) in floxed control islets (Figure 4a). Secondly, we loaded isolated islets with $\mathrm{H}_{2} \mathrm{DCF}$, a nonfluorescent precursor that is converted in excitable DCF by oxidation proportionally to the amount of intracellular free radicals. This assay revealed an increased amount of ROS formation in knockout islets compared with floxed control islets (Figure 4b). Taken together, the results indicate that disruption of frataxin in pancreatic $\beta$ cells leads to increased formation of ROS, which (based on the ESR spectra) consist mainly of superoxide. Whether this leads unambiguously to the phenotype observed rather than being of coincidental relevance remains to be evaluated.

Frataxin-deficient islets show decreased proliferation and increased apoptosis. To investigate effects of frataxin gene disruption and possibly ROS on islet proliferation and/or apoptosis, immunohistochemical methods were used. The rate of programmed cell death was shown to be significantly increased in sections from knockout pancreata, based on the number of islet cells immunopositive for activated caspase-3 (Figure 4c), a marker for apoptosis. Independently, reduced proliferation of $\beta$ cells was observed in islets from knockout animals, indicated by a significant decrease in number of $\beta$ cells immunopositive for the marker protein Ki67 and BrdU (Figure 4c). In summary, we conclude that the progressive decline in $\beta$ cell mass might be explained by both induction of apoptosis and a decreased regenerative capacity within pancreatic islets following disruption of the frataxin gene.

\section{Discussion}

Diabetes mellitus is caused by an absolute (type 1 ) or relative (type 2 ) deficiency of insulin-producing $\beta$ cells. Recent data suggest that in human type 2 diabetes increased apoptosis in pancreatic islets might be the underlying mechanism (33). It appears crucial for both prevention and treatment of the disease to understand the mechanism by which $\beta$ cell mass and function deteriorate. By disrupting the frataxin gene in pancreatic $\beta$ cells, we have engineered an animal model of slowly progressive reduction of islet mass and number. This reduction causes an impaired insulin-secretory response to glucose and carbohydrates and cumulates in overt diabetes. In theory, impaired stimulus-secretion coupling might be responsible for this impairment, and it was observed in a model for mitochondrial diabetes (31). In contrast, we here observe unaltered insulin secretion from isolated islets regardless of the phenotype, suggesting that stimulus-secretion coupling is intact in frataxin-deficient $\beta$ cells, consistent with findings in frataxin-deficient humans (see Introduction). On the other hand, mice at 6 weeks of age exhibit a somewhat impaired glucose tolerance (Figure $3 c)$, though the area under the curve for this particular test was not significantly different at that age $(P=0.0647)$. Furthermore, very young knockout animals exhibit lower postprandial glucose excursions (Figure 2a), suggesting hypersecretion, as observed in early prediabetes in humans (34). Hence we cannot rule out a minor influence of impaired stimulus-secretion coupling following frataxin knockout in pancreatic $\beta$ cells regardless of the ex vivo experiments. Nevertheless, since development of overt diabetes requires 9 months in the present model and is preceded by altered islet growth characteristics followed by slowly progressing glucose intolerance, it appears evident that a degenerative process is responsible for the major phenotype observed. $\beta$ Cells of frataxin knockout mice exhibit increased apoptosis and a reduced islet-proliferation rate. While this mechanism clearly leads to diabetes in this particular model, numerous underlying pathways might be responsible for the observed impairment of secretory capacity. Frataxin has been shown primarily to direct synthesis of iron-sulphur clusters within mitochondria (5). Secondarily, lack of frataxin causes increased levels of ROS both in vivo $(7,8)$ and in vitro $(11$, 12). $\beta$ Cells are exceptionally sensitive to ROS (35). Additionally, hyperglycemia, the phenotypical hallmark of any subtype of diabetes, has been shown in vitro to induce formation of ROS and specifically superoxide in insulin-secreting cells $(36,37)$ and vascular endothelial cells (38). Finally, $\beta$ cells react to hyperglycemia by induction of both antiapoptotic and antioxidant genes (39). We here demonstrate that disruption of frataxin leads to increased levels of ROS within pancreatic islets, which in turn are associated with progressive reduction of insulin-secretory capacity. Of note, in our model, ROS formation precedes both hyperglycemia and impaired insulin-secretory capacity, 
while it is paralleled by increased apoptosis and decreased proliferation. Hence it appears feasible that ROS formation in islets from frataxin knockout animals might be responsible for reduction of islet mass, number, and relative size. We have initiated further studies using targeted antioxidants to seek compelling evidence for this hypothesis. It should be noted, though it was not investigated in this study because of restriction of the knockout to pancreatic $\beta$ cells, that humans with reduced levels of frataxin protein exhibit some degree of insulin resistance $(14,15)$. This suggests that there might be an additional role for frataxin in mediating insulin signaling, as further supported by in vitro evidence (6). Interestingly, insulin resistance has been independently associated with ROS formation in various models (40). Therefore, lack of frataxin might be responsible for the induction of diabetes by affecting both insulin signaling and insulin secretion; the latter is demonstrated in the present study.

Together, the data indicate that disruption of frataxin in pancreatic $\beta$ cells causes slowly progressive diabetes in mice due to reduced islet mass preceded by increased formation of ROS, increased apoptosis, and decreased proliferation of pancreatic $\beta$ cells.

\section{Acknowledgments}

The expert technical assistance of Swetlana König, Ute Lehmann, Elisabeth Meyer, Susann Richter, and Elke Thom is gratefully acknowledged. Parts of this work were supported by grants from the Deutsche Forschungsgemeinschaft (to M. Ristow), the FritzThyssen-Stiftung (to M. Ristow), the Deutsche Diabetes-Gesellschaft (to M. Ristow), the Deutsche Diabetes-Stiftung (to M. Ristow), and the Juvenile Diabetes Foundation (to H. Mulder).

1. Friedreich, N. 1863. Ueber degenerative Atrophie der spinalen Hinterstränge. Virchows Archiv der Pathologischen Anatomie. 26:391-419.

2. McKusick, V.A., et al. 2003. Friedreich ataxia. www.ncbi.nlm.nih.gov/ htbin-post/Omim/dispmim?229300.

3. Campuzano, V., et al. 1996. Friedreich's ataxia: autosomal recessive disease caused by an intronic GAA triplet repeat expansion. Science. 271:1423-1427.

4. Koutnikova, H., et al. 1997. Studies of human, mouse and yeast homologues indicate a mitochondrial function for frataxin. Nat. Genet. 16:345-351.

5. Mühlenhoff, U., Richhardt, N., Ristow, M., Kispal, G., and Lill, R. 2002. The yeast frataxin homologue Yfh $1 \mathrm{p}$ plays a specific role in the maturation of cellular Fe/S proteins. Hum. Mol. Genet. 11:2025-2036.

6. Ristow, M., et al. 2000. Frataxin activates mitochondrial energy conversion and oxidative phosphorylation. Proc. Natl. Acad. Sci. U. S. A. 97:12239-12243.

7. Schulz, J.B., et al. 2000. Oxidative stress in patients with Friedreich ataxia. Neurology. 55:1719-1721.

8. Emond, M., Lepage, G., Vanasse, M., and Pandolfo, M. 2000. Increased levels of plasma malondialdehyde in Friedreich ataxia. Neurology. 55:1752-1753.

9. Cossee, M., et al. 2000. Inactivation of the Friedreich ataxia mouse gene leads to early embryonic lethality without iron accumulation. Hum. Mol. Genet. 9:1219-1226.

10. Puccio, H., et al. 2001. Mouse models for Friedreich ataxia exhibit cardiomyopathy, sensory nerve defect and Fe-S enzyme deficiency followed by intramitochondrial iron deposits. Nat. Genet. 27:181-186.

11. Chantrel-Groussard, K., et al. 2001. Disabled early recruitment of antioxidant defenses in Friedreich's ataxia. Hum. Mol. Genet. 10:2061-2067.

12. Shoichet, S.A., et al. 2002. Frataxin promotes antioxidant defense in a thiol-dependent manner resulting in diminished malignant transformation in vitro. Hum. Mol. Genet. 11:815-821.

13. Finocchiaro, G., Baio, G., Micossi, P., Pozza, G., and di Donato, S. 1988. Glucose metabolism alterations in Friedreich's ataxia. Neurology. 38:1292-1296

14. Khan, R.J., Andermann, E., and Fantus, I.G. 1986. Glucose intolerance in Friedreich's ataxia: association with insulin resistance and decreased insulin binding. Metabolism. 35:1017-1023.

15. Hebinck, J., et al. 2000. Heterozygous expansion of the GAA tract of the $\mathrm{X} 25$ /frataxin gene is associated with insulin resistance in humans. Diabetes. 49:1604-1607.

16. Ristow, M., et al. 1998. An association between NIDDM and a GAA trinucleotide repeat polymorphism in the X25/frataxin (Friedreich's ataxia) gene. Diabetes. 47:851-854.

17. Dupont, S., et al. 1998. No association between the Friedreich's ataxia gene and NIDDM in the French population. Diabetes. 47:1654-1656.

18. Dalgaard, L.T., et al. 1999. Intermediate expansions of a GAA repeat in the frataxin gene are not associated with type 2 diabetes or altered glucose-induced beta-cell function in Danish Caucasians. Diabetes. 48:914-917.

19. 't Hart, L.M., et al. 1999. Altered beta-cell characteristics in impaired glucose tolerant carriers of a GAA trinucleotide repeat polymorphism in the frataxin gene. Diabetes. 48:924-926.

20. Lynn, S., et al. 2000. Intermediate expansions of a X25/frataxin gene GAA repeat and type II diabetes: assessment using parent-offspring trios. Diabetologia. 43:384-385.

21. Shadrina, M.I., Miloserdova, O.V., Slominskii, P.A., Balabolkin, M.I., and Limborskaya, S.A. 2002. Association of polymorphic trinucleotide repeat (GAA)n of the frataxin gene with diabetes mellitus type 2 in the Moscow population. Mol. Biol. (Mosk.). 36:37-39.

22. Hanis, C.L., et al. 1996. A genome-wide search for human non-insulindependent (type 2) diabetes genes reveals a major susceptibility locus on chromosome 2. Nat. Genet. 13:161-166.

23. Pratley, R.E., et al. 1998. An autosomal genomic scan for loci linked to prediabetic phenotypes in Pima Indians. J. Clin. Invest. 101:1757-1764.

24. Luo, T.H., et al. 2001. A genome-wide search for type II diabetes susceptibility genes in Chinese Hans. Diabetologia. 44:501-506.

25. Lindgren, C.M., et al. 2002. Genomewide search for type 2 diabetes mellitus susceptibility loci in Finnish families: the Botnia study. Am.J. Hum. Genet. 70:509-516.

26. Accili, D., Kido, Y., Nakae, J., Lauro, D., and Park, B.C. 2001. Genetics of type 2 diabetes: insight from targeted mouse mutants. Curr. Mol. Med. 1:9-23.

27. Kulkarni, R.N., et al. 1999. Tissue-specific knockout of the insulin receptor in pancreatic beta cells creates an insulin secretory defect similar to that in type 2 diabetes. Cell. 96:329-339.

28. Lacy, P.E., and Kostianovsky, M. 1967. Method for the isolation of intact islets of Langerhans from the rat pancreas. Diabetes. 16:35-39.

29. Bradford, M.M. 1976. A rapid and sensitive method for the quantitation of microgram quantities of protein utilizing the principle of protein-dye binding. Anal. Biochem. 72:248-254.

30. Postic, C., et al. 1999. Dual roles for glucokinase in glucose homeostasis as determined by liver and pancreatic beta cell-specific gene knockouts using cre recombinase. J. Biol. Chem. 274:305-315.

31. Silva, J.P., et al. 2000. Impaired insulin secretion and beta-cell loss in tissue-specific knockout mice with mitochondrial diabetes. Nat. Genet. 26:336-340.

32. Ke, L.D., Chen, Z., and Yung, W.K. 2000. A reliability test of standardbased quantitative PCR: exogenous vs endogenous standards. Mol. Cell. Probes. 14:127-135.

33. Butler, A.E., et al. 2003. Beta-cell deficit and increased beta-cell apoptosis in humans with type 2 diabetes. Diabetes. 52:102-110.

34. Hofeldt, F.D. 1989. Reactive hypoglycemia. Endocrinol. Metab. Clin. North Am. 18:185-201.

35. Tiedge, M., Lortz, S., Drinkgern, J., and Lenzen, S. 1997. Relation between antioxidant enzyme gene expression and antioxidative defense status of insulin-producing cells. Diabetes. 46:1733-1742.

36. Ihara, Y., et al. 1999. Hyperglycemia causes oxidative stress in pancreatic beta-cells of GK rats, a model of type 2 diabetes. Diabetes. 48:927-932.

37. Kaneto, H., et al. 2001. Activation of the hexosamine pathway leads to deterioration of pancreatic beta-cell function through the induction of oxidative stress. J. Biol. Chem. 276:31099-31104.

38. Nishikawa, T., et al. 2000. Normalizing mitochondrial superoxide production blocks three pathways of hyperglycaemic damage. Nature. 404:787-790.

39. Laybutt, D.R., et al. 2002. Increased expression of antioxidant and antiapoptotic genes in islets that may contribute to beta-cell survival during chronic hyperglycemia. Diabetes. 51:413-423.

40. Evans, J.L., Goldfine, I.D., Maddux, B.A., and Grodsky, G.M. 2003. Are oxidative stress-activated signaling pathways mediators of insulin resistance and beta-cell dysfunction? Diabetes. 52:1-8. 